\title{
Análise biomecânica de dois tipos de fixação de fratura supracondiliana de úmero em modelo anatômico*
}

\section{Biomechanical Analysis of Two Types of Humerus Supracondylar Fracture Fixation in Anatomical Model}

\author{
Marcos Ceita Nunes ${ }^{10}$ Ticiano Dozza Posser ${ }^{1} \quad$ Charles Leonardo Israel ${ }^{2}$ Leandro de Freitas Spinelli ${ }^{2,3}$ \\ Luis Gustavo Calieron ${ }^{1}$ Jung Ho Kim ${ }^{1}$
}

1 Serviço de Ortopedia Pediátrica, Hospital São Vicente de Paulo, Passo Fundo, RS, Brasil

Address for correspondence Marcos Ceita Nunes, MD, Rua Uruguai,

${ }^{2}$ Laboratório de Bioengenharia, Universidade de Passo Fundo, Passo 2050, Passo Fundo, RS, Brasil. Cep: 99010-112

Fundo, RS, Brasil

(e-mail: marcosceitanunes@yahoo.com.br; pesquisa2@hsvp.com.br).

${ }^{3}$ Serviço de Ortopedia e Traumatologia, Hospital Santa Casa de

Misericórdia de Porto Alegre, Porto Alegre, RS, Brasil

Rev Bras Ortop 2019;54:261-267.

\section{Resumo}

Palavras-chave

- fenômenos biomecânicos

- epífises/lesões

- fixação de fratura

- fraturas do úmero
Objetivo Analisar através de ensaios mecânicos a estabilidade da fixação da fratura supracondiliana do úmero com dois fios de Kirschner, intramedular e lateral (Fi), comparada à fixação com dois fios laterais paralelos (FL) em modelos anatômicos, de forma a se definir qual configuração apresenta maior estabilidade.

Métodos Foram utilizados como corpos de prova 72 úmeros sintéticos, os quais foram seccionados transversalmente para simular a fratura. Estes ossos foram divididos em dois grupos iguais e as fraturas fixadas com dois fios de Kirschner paralelos (FL) e com um fio lateral e outro intramedular (Fi). Então os corpos de prova foram submetidos aos testes de carga em estresse em uma máquina de ensaio universal, medidos em Newtons (N). Cada grupo foi subdividido em carga em varo, em valgo, em extensão, em flexão, em rotação externa e em rotação interna. A análise dos dados foi realizada comparando os subgrupos do grupo FL, com seus respectivos subgrupos do grupo Fi através do teste t bicaudal.

Resultados $O$ teste $t$ bicaudal demonstrou que em 4 das 6 condições aplicadas não houve diferença estatística significativa entre os grupos $(p>0,05)$. Encontramos uma diferença significativa entre os grupos com carga em extensão com uma média das maiores forças no grupo FL de $19 \mathrm{~N}$ e no grupo Fi de $28,7 \mathrm{~N}(p=0,004)$, e também entre os grupos com carga em flexão com a média de forças registradas no grupo FL de $17,1 \mathrm{~N}$ e no grupo Fi de $22,9 \mathrm{~N}(p=0,01)$.

Conclusão A fixação com fio intramedular e um fio lateral para cargas em extensão e flexão apresenta maior estabilidade quando comparada com a fixação com dois fios laterais paralelos, sugerindo resultados clínicos no mínimo semelhantes.

Trabalho realizado no Hospital São Vicente de Paulo, Passo Fundo, RS, Brasil.

(1D) Marcos Ceita Nunes's ORCID is https://orcid.org/0000-00022307-8359.

received

September 28, 2017

accepted

July 2, 2018
DOI https://doi.org/

10.1055/s-0039-1688756. ISSN 0102-3616.
Copyright $(\odot 2019$ by Sociedade Brasileira License terms de Ortopedia e Traumatologia. Published by Thieme Revnter Publicações Ltda, Rio de Janeiro, Brazil 


\begin{abstract}
Keywords

- biomechanical phenomena

- epiphyses/injuries

- fracture fixation

- humeral fractures

Objective To analyze the stability of humerus supracondylar fracture fixation with Kirschner wires comparing intramedullary and lateral ( $\mathrm{Fi})$, and two parallel lateral wires (FL) fixation in experimental models, to define which configuration presents greater stability.

Methods A total of 72 synthetic humeri were cross-sectioned to simulate the fracture. These bones were divided into two equal groups and the fractures were fixed with parallel Kirschner wires (FL) and with a lateral and intramedullary (Fi) wire. Then, the test specimens were subjected to stress load tests on a universal test machine, measured in Newtons (N). Each group was subdivided into varus load, valgus, extension, flexion, external rotation and internal rotation. An analysis of the data was performed comparing the subgroups of the FL group with their respective subgroups of the Fi group through the two-tailed $t$ test.

Results The two-tailed t test showed that in 4 of the 6 evaluated conditions there was no significant statistical difference between the groups $(p>0.05)$. We have found a significant difference between the group with extension load with a mean of $19 \mathrm{~N}$ (FL group) and of $28.7 \mathrm{~N}$ (Fi group) $(p=0.004)$, and also between the groups with flexural load with the mean of the forces recorded in the FL group of $17.1 \mathrm{~N}$ and of $22.9 \mathrm{~N}$ in the Fi group $(p=0.01)$.

Conclusion Fixation with one intramedullary wire and one lateral wire, considering loads in extension and flexion, presents greater stability when compared to a fixation with two lateral wires, suggesting similar clinical results.
\end{abstract}

\section{Introdução}

A fratura supracondiliana é mais comum no período entre 4 a 7 anos de idade, ${ }^{1}$ corresponde a dois terços das crianças hospitalizadas por trauma em cotovelo e a $30 \%$ das fraturas nesta faixa etária. ${ }^{2}$ Devido à anatomia particular do cotovelo, a configuração das partes moles e a ordem de ossificação dos núcleos de crescimento, a fratura supracondiliana se comporta em padrões quase sempre definidos em extensão, com deslocamentos posteromediais, posterolaterais e em flexão. O grau de deslocamento é definido pela direção da força deformante, pela posição do membro na hora do trauma e pela magnitude desta força. ${ }^{3,4}$

Gartland classificou esta fratura em três tipos de acordo com o grau de deslocamento: é consenso que as fraturas de grau 1 são de tratamento conservador. ${ }^{5}$ Existem também artigos descrevendo técnicas conservadoras com redução e imobilização nas de grau 2 e $3 .^{6}$ Porém, muitos autores descrevem a redução e fixação percutânea como o padrão ouro nas fraturas deslocadas. ${ }^{7,8}$ Neste sentido, não existe consenso quanto à melhor posição dos fios de Kirschner na estabilização desta fratura. ${ }^{9}$ A fixação com configuração com fios cruzados dá uma melhor estabilidade; no entanto, há o risco de lesão iatrogênica do nervo ulnar. A configuração com dois fios laterais demonstrou ter estabilidade inferior aos fios cruzados, menor incidência de lesões do nervo ulnar e ser tecnicamente mais difícil, pois tanto na direção divergente quanto na paralela o espaço lateral para colocação dos fios é diminuto. Entretanto, ambas apresentam resultados clínicos semelhantes. ${ }^{10-13}$

Em 1991, Bertol et al ${ }^{14}$ publicaram a técnica de fixação das fraturas supracondilianas com desvio posteromedial com um fio de Kirschner intramedular inserido logo lateral ao olécrano e outro lateral com entrada no epicôndilo, sendo esta técnica presumidamente mais fácil, pois otimiza o espaço da coluna lateral.

Existem inúmeros estudos biomecânicos comparando as diversas configurações de posição dos fios de Kirchner na estabilização da fratura supracondiliana de úmero, ${ }^{13,15,16}$ porém não existe nenhum estudo que analise a configuração com um fio intramedular e outro lateral. O objetivo do presente estudo é comparar a técnica de fixação com fio intramedular com a técnica de fixação com dois fios paralelos laterais.

\section{Materiais e Métodos}

A presente pesquisa utilizou como corpos de prova 72 úmeros esquerdos sintéticos com canal medular e material esponjoso modelo 3022B (Nacional Ossos, Jaú, SP, Brasil) que foram seccionados igualmente de forma transversal, paralelamente à superfície articular no plano coronal, com uma serra guiada em sua porção distal, com a secção passando na fossa do olécrano a $3 \mathrm{~cm}$ da borda distal do úmero, simulando uma fratura supracondiliana (-Fig. 1). Optamos pelo corte transversal, pois $80 \%$ das fraturas supracondilianas apresentam um padrão de fratura transversal na radiografia em perfil ${ }^{17} \mathrm{e}$, além disso, a obliquidade da fratura determina instabilidade. ${ }^{18}$

Os úmeros sintéticos seccionados foram divididos em dois grupos conforme a configuração da fixação: grupo com fixação com dois fios laterais (FL) e grupo com fixação com um fio intramedular e um fio lateral (Fi). Todos os modelos tiveram redução anatômica e fixação com fios de Kirschner 

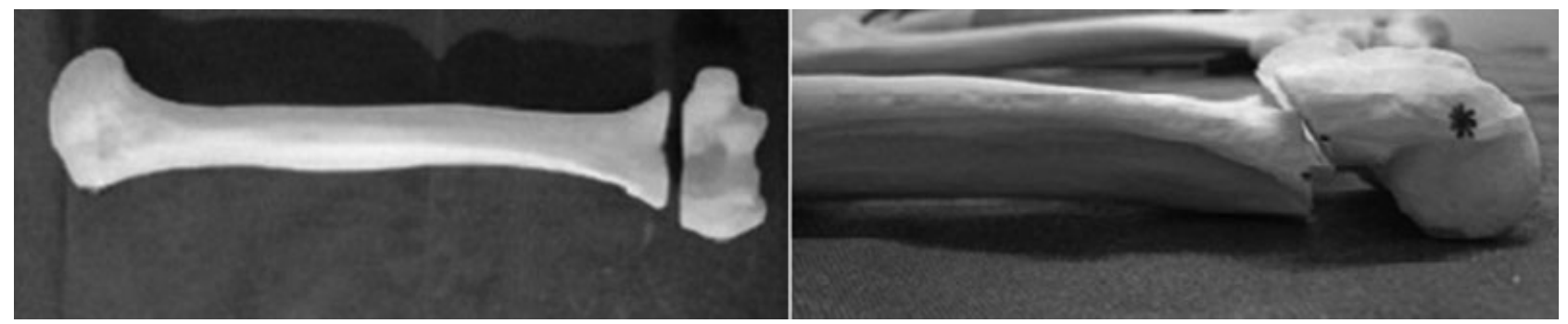

Fig. 1 Corpos de prova: úmeros sintéticos seccionados transversalmente em sua porção distal, com a secção passando na fossa do olécrano a $3 \mathrm{~cm}$ da borda distal do úmero, simulando uma fratura supracondiliana.

$2.0 \mathrm{~mm}$. Confeccionamos um modelo padronizado de fixação para cada grupo, assegurando que as fixações de cada grupo fossem idênticas. No grupo FL, a fixação foi realizada com dois fios de Kirschner $2.0 \mathrm{~mm}$ com entrada no epicôndilo lateral, sendo o fio mais distal na borda inferior do epicôndilo lateral e o fio proximal $1 \mathrm{~cm}$ acima, paralelos, a $30^{\circ} \mathrm{com}$ o eixo da diáfise do úmero, fixados na cortical oposta $3 \mathrm{~cm}$ acima da linha de fratura.

No grupo Fi, a fixação também foi realizada com dois fios de Kirschner $2.0 \mathrm{~mm}$ com o $1^{\circ}$ fio entrando $2 \mathrm{~mm}$ lateral à borda lateral da tróclea, no sulco capítulo-troclear, ficando intramedular, sendo introduzido até a transição do terço médio com o terço distal do úmero a $11 \mathrm{~cm}$ da extremidade distal do úmero, e o $2^{\circ}$ fio introduzido no centro do epicôndilo lateral, a $30^{\circ}$ com o eixo da diáfise do úmero, cruzando o primeiro fio a $2 \mathrm{~cm}$ da linha de fratura e fixado na cortical oposta a $3 \mathrm{~cm}$ da linha de fratura.

As fixações foram feitas com auxilio de um perfurador e de fluoroscopia. Todos os corpos de prova foram comparados com seus respectivos modelos padronizados através de fluoroscopia, seguindo os critérios de fixação já citados, assegurando a semelhança entre eles (-Fig. 2).
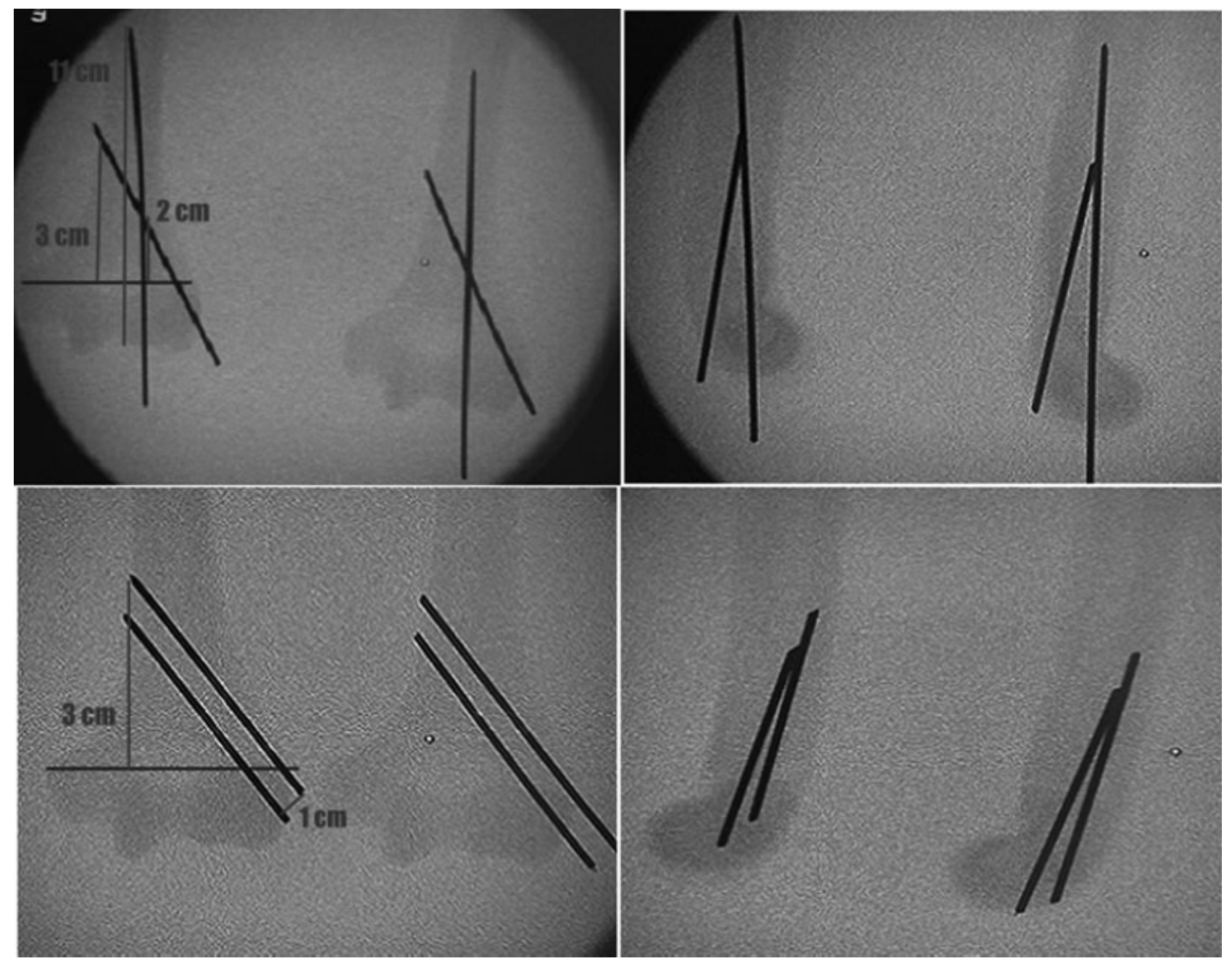

Fig. 2 Corpos de prova comparados com seus respectivos modelos padronizados através de fluoroscopia, seguindo os critérios de fixação e assegurando a semelhança entre eles. 
Os corpos de prova que não apresentavam os critérios de fixação foram excluídos. Após a fixação, os úmeros de cada grupo foram encaminhados ao Laboratório de Engenharia, onde, em conjunto com um engenheiro colaborador, separamos cada grupo em subgrupos conforme os testes de carga aos quais foram submetidos: subgrupo 1: carga em varo; subgrupo 2: carga em valgo; subgrupo 3: carga em extensão; subgrupo 4: carga em flexão; subgrupo 5: carga em rotação interna; e subgrupo 6: carga em rotação externa.

Os testes de carga foram realizados em uma máquina de ensaio universal de tração modelo UPM 200 (3022B, left humerus with medullary canal and spongy material, Nacional Ossos, Jaú, SP, Brazil) e uma célula de carga HBM U9B (3022B, left humerus with medullary canal and spongy material, Nacional Ossos, Jaú, SP, Brazil) $(20 \mathrm{KN}=1 \mathrm{mV} / \mathrm{V})$. Esta mede a carga gerada em Newtons (N) durante deslocamento contínuo promovido pela máquina de ensaio de tração a uma velocidade de $1 \mathrm{~mm} /$ segundo, com um deslocamento máximo estabelecido de $10 \mathrm{~cm}$, o que promove uma angulação de até $45^{\circ}$ no corpo de prova, com fulcro de rotação no traço de fratura (-Fig. 3 ).

Foi desenvolvido um suporte para acoplar anatomicamente o úmero distal, permitindo que as cargas fossem aplicadas e mensuradas nos modelos de ossos, em um ponto $10 \mathrm{~cm}$ proximal ao traço de fratura, até que atingissem $45^{\circ}$ de angulação e/ou quebra do material ( - Fig. 4). Também foi elaborado um mecanismo para criar forças rotacionais a partir da carga estabelecida pela máquina de ensaio de tração (- Fig. 5). As cargas rotacionais foram aplicadas até a quebra dos modelos ósseos.

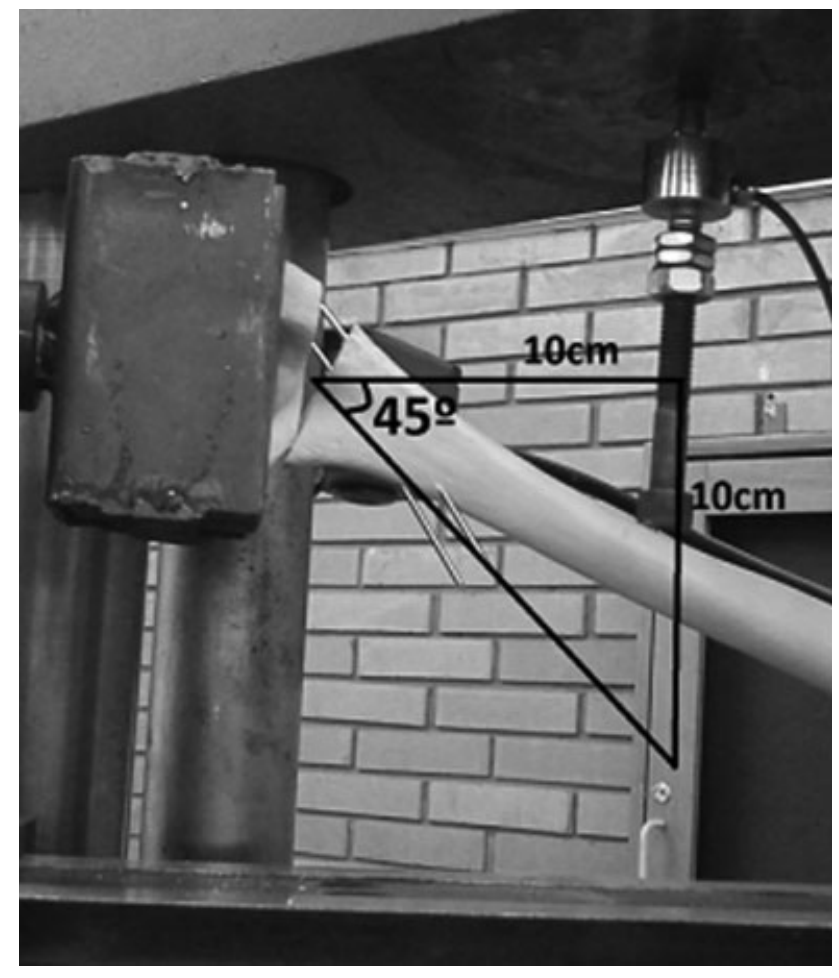

Fig. 4 Cargas aplicadas e mensuradas nos corpos de prova, em um ponto $10 \mathrm{~cm}$ proximal ao traço de fratura, até que atingissem $45^{\circ}$ de angulação e/ou quebra do material.
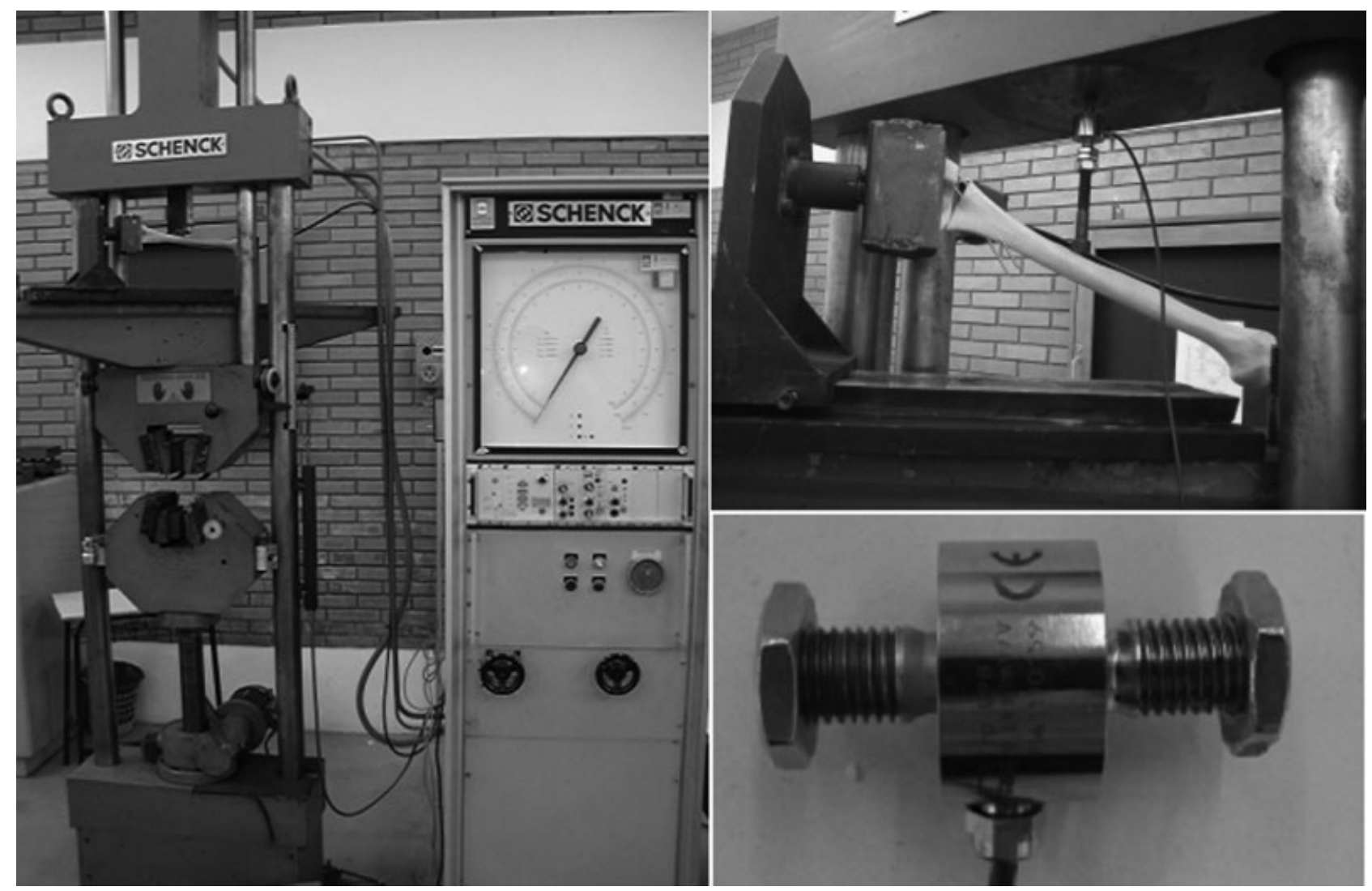

Fig. 3 Máquina de ensaio universal de tração modelo UPM 200 e uma célula de carga HBM U9B (20 KN = 1 mV/V). 


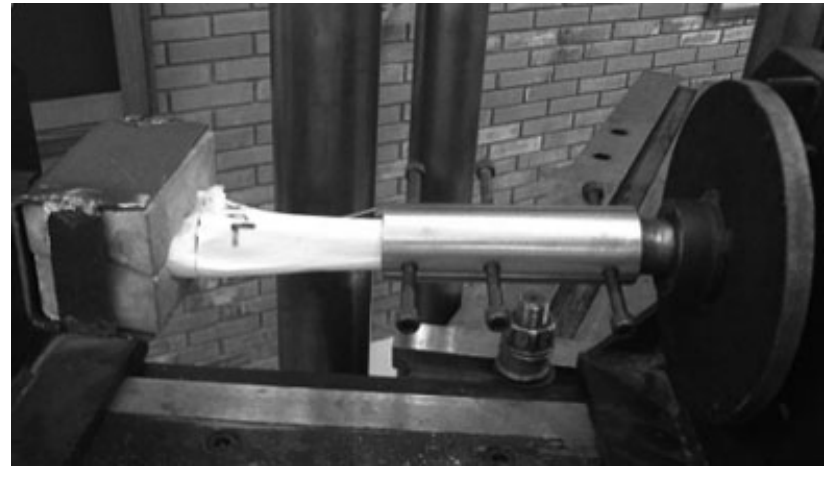

Fig. 5 Mecanismo para aplicação das cargas torcionais.

Durante a análise dos dados de cada modelo ósseo, gerados pela célula de carga, observamos que, durante o período de deslocamento estabelecido, a força, registrada em $\mathrm{N}$, apresenta inicialmente um caráter ascendente, até atingir um platô, este relacionado com as maiores forças registradas. Após o platô, ocorre uma diminuição da força aplicada que está relacionada com a quebra do modelo ósseo e/ou $45^{\circ}$ de deslocamento. Dessa forma, definimos a força em $\mathrm{N}$ ao atingir este platô como a variável a ser analisada, ou seja, a força máxima registrada durante o deslocamento, ao final da região linear do gráfico.

O cálculo do tamanho da amostra foi realizado no programa PEPI (Programs for Epidemiologists) versão 4.0 e baseado no estudo de Bloom et al. ${ }^{19}$ Para um nível de significância de $5 \%$, um poder de $90 \%$, um desvio padrão (DP) estimado em 3,5 N com uma diferença de médias de $8 \mathrm{~N}$, obteve-se um total mínimo de 6 peças por subgrupo, totalizando 36 por grupo.

A análise dos dados foi realizada através do programa Microsoft Office Excel 2010 (Microsoft Corporation, Redmond, WA, EUA), comparando os subgrupos do grupo FL, com seus respectivos subgrupos do grupo Fi através do teste t bicaudal. $O$ presente estudo não apresenta conflito de interesses.

\section{Resultados}

Os resultados dos testes de carga para comparar a estabilidade das duas configurações de fios em questão estão representados em $\mathrm{N}$ na - Tabela 1. O teste $\mathrm{t}$ bicaudal demonstrou que em 4 das 6 cargas aplicadas não houve diferença estatística significativa $(p<0,05)$ ( $>$ Tabela 1 ).

Nos modelos ósseos submetidos à carga em varo obtivemos uma média das maiores forças registradas durante 0 deslocamento no grupo FL de $28,7 \mathrm{~N}$ e DP $=3,5 \mathrm{~N}$. No grupo Fi, encontramos uma média de $30,7 \mathrm{~N}$ e DP $=4,9 \mathrm{~N}$. Desta forma, o teste $\mathrm{t}$ bicaudal não revelou uma diferença estatística significativa entre os grupos ( $p=0,23$ ) (-Fig. 6).

Nos modelos submetidos à carga em valgo, a média das maiores forças registradas no grupo $\mathrm{FL}$ foi de $20,6 \mathrm{~N}$ e $\mathrm{DP}=5,2 \mathrm{~N}$. No grupo $\mathrm{Fi}$, encontramos uma média de $22,9 \mathrm{~N}$ e DP $=3,4 \mathrm{~N}$. Assim como na carga em varo, o teste $\mathrm{t}$ bicaudal não revelou uma diferença estatística significativa

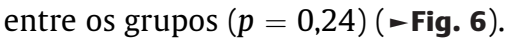

Também não encontramos uma diferença estatística significativa entre os grupos nos testes de carga em rotação
Tabela 1 Dados de força e direção da carga mecânica

\begin{tabular}{|l|l|l|l|}
\hline & Grupo FL & Grupo Fi & $\begin{array}{l}\text { Teste t } \\
\text { bicaudal }\end{array}$ \\
\hline Varo $(n)$ & $28,7 \pm 3,5$ & $30,7 \pm 4,9$ & $p=0,230$ \\
\hline Valgo $(n)$ & $20,6 \pm 5,2$ & $22,9 \pm 3,4$ & $p=0,240$ \\
\hline Extensão $(n)$ & $19,0 \pm 3,4$ & $28,7 \pm 4,0$ & $p=0,004$ \\
\hline Flexão $(n)$ & $17,1 \pm 1,2$ & $22,9 \pm 4,0$ & $p=0,015$ \\
\hline $\begin{array}{l}\text { Rotação } \\
\text { Interna }(n)\end{array}$ & $12,55 \pm 1,2$ & $11,7 \pm 2,6$ & $p=0,256$ \\
\hline $\begin{array}{l}\text { Rotação } \\
\text { Externa }(n)\end{array}$ & $11,2 \pm 1,8$ & $11,6 \pm 1,0$ & $p=0,292$ \\
\hline
\end{tabular}

Abreviações: Fi, fixação com um fio intramedular e um fio lateral; FL, fixação com dois fios laterais.

interna e externa ( $p=0,25$ e $p=0,24$, respectivamente) (-Fig. 6).

Obtivemos uma diferença estatisticamente significativa entre os 2 grupos nos testes de carga em extensão $(p=0,004)$, com uma média das maiores forças registradas no grupo FL de 19,0 N, DP = 3,4 N, e no grupo Fi de 28,7 N, $\mathrm{DP}=4,0 \mathrm{~N}$ (-Fig. 6). Desta forma, durante o deslocamento constante estabelecido pela máquina de ensaio foi gerada, e registrada pela célula de carga, uma força maior no grupo $\mathrm{Fi}$ comparado ao grupo FL. Assim, também podemos sugerir uma maior estabilidade na configuração com um fio intramedular e um fio lateral para cargas em extensão quando comparada a configuração com dois fios laterais paralelos.

Os modelos submetidos à carga em flexão também revelaram uma diferença estatística significativa entre os grupos $(p=0,01)$, sendo que a média das maiores forças registradas no grupo FL foi de $17,1 \mathrm{~N}$, com $\mathrm{DP}=1,2 \mathrm{~N}$, e no grupo $\mathrm{Fi}$, de 22,9 N, com DP $=4 \mathrm{~N}$ (-Fig. 6).

\section{Discussão}

O principal objetivo do tratamento das fraturas supracondilianas deslocadas é obter uma redução anatômica e conseguir uma fixação segura evitando deformidades angulares. Isso geralmente é conseguido com redução fechada e fixação percutânea. $^{20-22}$ Para realizar a fixação, deve-se dar total atenção ao exame clínico e radiológico do cotovelo contralateral, obter verdadeiras projeções ortogonais na fluoroscopia e considerar parâmetros radiográficos bem descritos na literatura para obter total correção das deformidades. ${ }^{23}$ As consolidações viciosas estão relacionadas também a fixações inadequadas e a erros técnicos no momento das fixações. ${ }^{19}$

Diversos estudos biomecânicos têm demonstrado que a fixação com fios cruzados apresenta uma maior estabilidade rotacional do que as fixações com fios laterais, ${ }^{15}$ porém apresenta um maior risco de lesão iatrogênica do nervo ulnar. $^{12}$ Bloom et al ${ }^{16}$ relataram que três pinos divergentes laterais apresentam resistência equivalente a dois fios cruzados, os quais são mais resistentes a dois fios laterais. Porém, na maioria dos casos, não há espaço suficiente para a colocação de pinos laterais. ${ }^{19}$ Em um estudo clínico randomizado prospectivo comparando técnicas de fixação lateral e cruzada no 


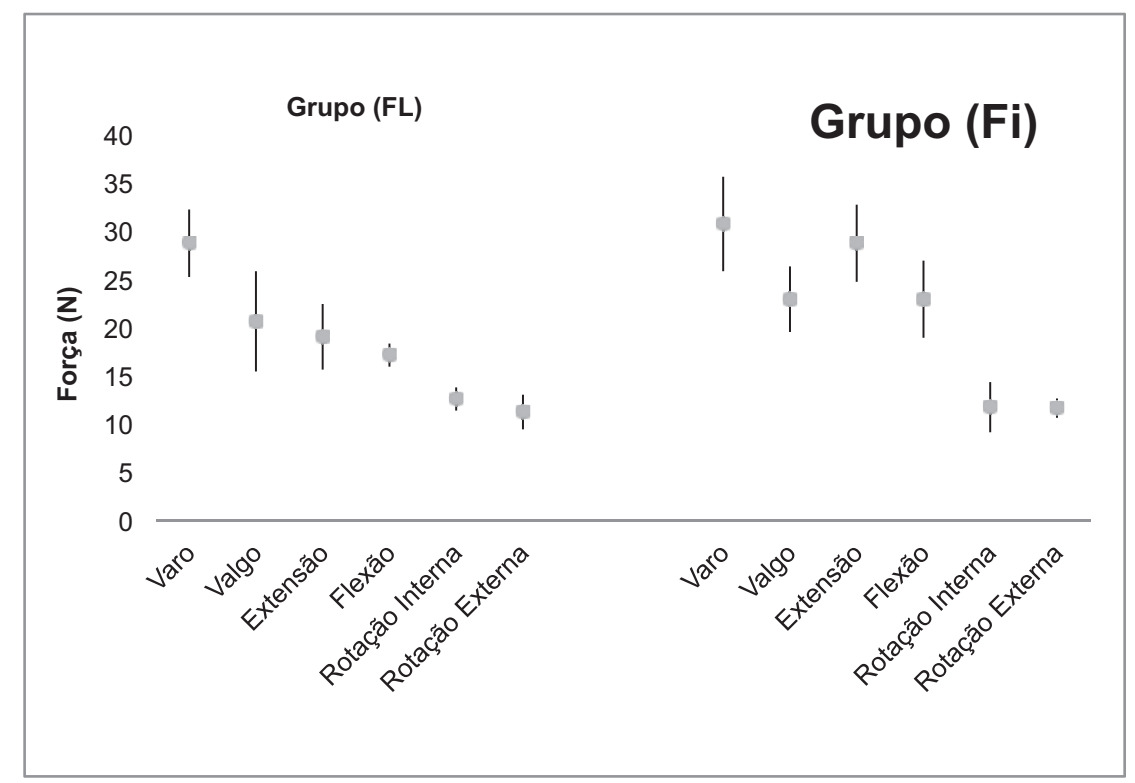

Fig. 6 Gráfico mostrando os resultados dos testes das cargas em varo, valgo, extensão e flexão e rotação interna e externa.

tratamento de fraturas supracondilianas de úmero tipo III, Kocher et al ${ }^{11}$ não encontraram diferença significativa entre os dois grupos com relação ao resultado radiográfico e clínico. Em outro estudo prospectivo randomizado, Blanco et $a^{24}$ não encontraram diferenças radiológicas significantes entre a fixação com fios cruzados e laterais.

Em nosso estudo, observamos que a técnica com fio intramedular apresenta uma resistência maior nas cargas em flexão e extensão do que a técnica com fios laterais, sendo as demais cargas semelhantes. Na técnica com fio intramedular, o primeiro passo após conseguir uma redução adequada é a introdução do fio intramedular, ${ }^{14}$ bloqueando as forças no sentido axial, principalmente flexão e extensão, permitindo de uma forma segura que as deformidades rotacionais possam ainda ser corrigidas, ou seja, esta técnica permite um ajuste rotacional após uma redução precisa no sentido axial, o que não é possível com a fixação com fios laterais. Desta forma, é mais fácil ter uma redução anatômica com a fixação com fio intramedular, e a obtenção de uma redução anatômica maximiza a estabilidade de todas as configurações de fixação. ${ }^{19}$ Além disso, a fixação com fio intramedular mantém um maior espaço lateral para a colocação dos fios.

Como a técnica com dois fios cruzados apresenta resultados clínicos semelhantes à com dois fios laterais, ${ }^{1,24-26}$ podemos pressupor, de acordo com o presente estudo mecânico, que a fixação com fio intramedular apresenta resultados clínicos equivalentes às técnicas citadas, porém é necessário elaborar um estudo clinico randomizado para a confirmação desta afirmativa.

Algumas limitações do presente estudo devem ser reconhecidas. Embora o uso de modelos sintéticos para análise mecânica de técnicas de redução da fratura seja comum na literatura, estas investigações não consideram a variabilidade nos padrões de fratura nem a anatomia com o periósteo circundante, as quais podem contribuir para a estabilidade do fragmento. ${ }^{15,27}$ Além disso, as cargas fisiológicas que atuam no cotovelo são certamente mais complexas do que o único eixo das direções de carga de teste utilizado no presente estudo. Além disso, os pinos foram colocados em uma situação ideal, sem levar em conta a dificuldade de introdução dos pinos no intraoperatório, a qual não pode ser simulada. O delineamento do estudo atual não permite comparações diretas das cargas aplicadas nos modelos com ossos orgânicos, permitindo apenas a comparação entre as técnicas de fixação dessas fraturas.

\section{Conclusão}

No presente estudo, a fixação com fio intramedular apresenta uma maior estabilidade nas cargas em flexão e extensão quando comparada à fixação com fios laterais, sendo semelhante em relação às demais cargas aplicadas, sugerindo resultados clínicos aceitáveis, como já comprovado por Bertol et al. ${ }^{14}$ Desta forma, mostra-se uma ótima opção de configuração dos fios de Kirschner para o tratamento destas fraturas.

\section{Conflitos de Interesses}

Os autores declaram não haver conflitos de interesses.

\section{Referências}

1 Cheng JC, Shen WY. Limb fracture pattern in different pediatric age groups: a study of 3,350 children. J Orthop Trauma 1993;7 (01):15-22

2 Kasser JR, Beaty JH. Supracondylar fractures of the distal humerus. In: Beaty JH, Kasser JR, Wilkins KE, Rockwood CE, eds. Rockwood and Wilkins' fractures in children. 6th ed. Philadelphia: Lippincott Williams and Wilkins; 2006:543-89

3 Omid R, Choi PD, Skaggs DL. Supracondylar humeral fractures in children. J Bone Joint Surg Am 2008;90(05):1121-1132

4 Mahan ST, May CD, Kocher MS. Operative management of displaced flexion supracondylar humerus fractures in children. J Pediatr Orthop 2007;27(05):551-556 
5 Barton KL, Kaminsky CK, Green DW, Shean CJ, Kautz SM, Skaggs DL. Reliability of a modified Gartland classification of supracondylar humerus fractures. J Pediatr Orthop 2001;21(01):27-30

6 Izadpanah M. [Closed treatment of supracondylar fractures of the humerus: a modification of Blounts technique (author's transl)]. Arch Orthop Unfallchir 1973;77(04):348-358

7 Wilkins K, Beaty J. Fractures in children. 4th ed. Philadelphia: Lippincott-Raven; 1996

8 Flynn JC, Zink WP. Fractures and dislocations of the elbow. In: MacEwen GD, Kasser JR, Heinrich SD, eds. Pediatric fractures. A practical approach to assessment and treatment. Baltimore: Willians \&Wilkins; 1993:133-64

9 Kasser JR, Beaty JH. Supracondylar fractures of the distal humerus. In: Beaty JH, Kasser JR, Wilkins KE, Rockwood CE, eds. Rockwood and Wilkins' fractures in children. 5th ed. Philadelphia: Lippincott Williams and Wilkins; 2004:594-95

10 Topping RE, Blanco JS, Davis TJ. Clinical evaluation of crossed-pin versus lateral-pin fixation in displaced supracondylar humerus fractures. J Pediatr Orthop 1995;15(04):435-439

11 Kocher MS, Kasser JR, Waters PM, Bae D, Snyder BD, Hresko MT, et al. Lateral entry compared with medial and lateral entry pin fixation for completely displaced supracondylar humeral fractures in children. A randomized clinical trial. J Bone Joint Surg Am 2007;89(04):706-712

12 Rasool MN. Ulnar nerve injury after K-wire fixation of supracondylar humerus fractures in children. J Pediatr Orthop 1998;18 (05):686-690

13 Brauer CA, Lee BM, Bae DS, Waters PM, Kocher MS. A systematic review of medial and lateral entry pinning versus lateral entry pinning for supracondylar fractures of the humerus. J Pediatr Orthop 2007;27(02):181-186

14 Bertol P, Monteggia GM, Paula MD. Fixação percutânea das fraturassupracondilianas do úmero na criança. Rev Bras Ortop 1991;26(03):48-51

15 Larson L, Firoozbakhsh K, Passarelli R, Bosch P. Biomechanical analysis of pinning techniques for pediatric supracondylar humerus fractures. J Pediatr Orthop 2006;26(05):573-578

16 Bloom T, Robertson C, Mahar A, Pring M, Newton PO. Comparison of supracondylar humerus fracture pinning when the fracture is not anatomically reduced. In: The Annual Meeting of the Pediatric Orthopaedic Society of North America, Hollywood, FL, 2007 May 23-26

17 Nand S. Management of supracondilar fractures in children. Int Surg 1972;177:203-209

18 Bahk MS, Srikumaran U, Ain MC, Erkula G, Leet AI, Sargent MC, et al. Patterns of pediatric supracondylar humerus fractures. J Pediatr Orthop 2008;28(05):493-499

19 Bloom T, Robertson C, Mahar AT, Newton P. Biomechanical analysis of supracondylar humerus fracture pinning for slightly malreduced fractures. J Pediatr Orthop 2008;28(07):766-772

20 Kurer MH, Regan MW. Completely displaced supracondylar fracture of the humerus in children. A review of 1708 comparable cases. Clin Orthop Relat Res 1990;(256):205-214

21 Royce RO, Dutkowsky JP, Kasser JR, Rand FR. Neurologic complications after K-wire fixation of supracondylar humerus fractures in children. J Pediatr Orthop 1991;11(02):191-194

22 Ito N, Eto M, Maeda K, Rabbi ME, Iwasaki K. Ultrasonographic measurement of humeral torsion. J Shoulder Elbow Surg 1995;4 (03):157-161

23 Pring M, Rang M, Wenger D. Elbow-distal humerus. In: Pring M, Rang M, Wenger D, eds. Rang's Children's Fractures. Philadelphia, PA: Lippincott Williams \& Wilkins; 2005:95-118

24 Blanco JS, Gaston G, Cates T, Busch MT, Schmitz ML, Schrader T, et al. Lateral pin versus crossed pin fixation in type 3 supracondylar humerus fractures: a randomized prospective study. In: The Annual Meeting of the Pediatric Orthopaedic Society of North America, Hollywood, FL, 2007 May 23-26.

25 Foead A, Penafort R, Saw A, Sengupta S. Comparison of two methods of percutaneous pin fixation in displaced supracondylar fractures of the humerus in children. J Orthop Surg (Hong Kong) 2004;12(01):76-82

26 Gordon JE, Patton CM, Luhmann SJ, Bassett GS, Schoenecker PL. Fracture stability after pinning of displaced supracondylar distal humerus fractures in children.J Pediatr Orthop 2001;21(03):313-318

27 Schwartz A, Oka R, Odell T, Mahar A. Biomechanical comparison of two different periarticular plating systems for stabilization of complex distal humerus fractures. Clin Biomech (Bristol, Avon) 2006;21(09):950-955 